\title{
A Hybrid Model of Sprouting Angiogenesis
}

\author{
Florian Milde, Michael Bergdorf, and Petros Koumoutsakos \\ Computational Science, ETH Zürich, CH-8092, Switzerland \\ petros@ethz.ch
}

\begin{abstract}
We present a computational model of tumor induced sprouting angiogenesis that involves a novel coupling of particle-continuum descriptions. The present 3D model of sprouting angiogenesis accounts for the effect of the extracellular matrix on capillary growth and considers both soluble and matrix-bound growth factors. The results of the simulations emphasize the role of the extracellular matrix and the different VEGF isoforms on branching behavior and the morphology of generated vascular networks.
\end{abstract}

Keywords: Blood vessel growth, Sprouting angiogenesis, Computational modeling, Particle-continuum coupling, 3D, Matrix-bound VEGF, Extracellular matrix, Branching.

\section{Introduction}

Sprouting angiogenesis, the process of new capillaries forming from existing vessels, can be observed in the human body under various conditions. In this work, we focus on tumor-induced angiogenesis, where a tumor in hypoxic conditions, secretes growth factors in order to establish its own vasculature to ensure nutrient and oxygen supply to the tumor cells leading to increased tumor cell proliferation and enhanced tumor growth.

The process of tumor-induced angiogenesis is initiated by tumor cells in conditions of glucose deprivation and hypoxia, with the shortage of oxygen supply triggering the release of angiogenic growth factors. Among the several growth factors known to contribute to the process, Vascular Endothelial Growth Factors (VEGF) have been identified as one of the key components. Upon release from the tumor, VEGFs diffuse through the ExtraCellular Matrix (ECM) occupying the space between tumor and existing vasculature and establish a chemical gradient. Once VEGF has reached a vessel, it binds to the receptors located on Endothelial Cells (EC), which line the blood vessel walls. This binding sets off a cascade of events triggering the outgrowth of new vessel sprouts at the existing vasculature near the tumor. While endothelial cell proliferation is confined to a region located behind the sprout tip, endothelial tip cells located at the sprouting front migrate through the ECM thus defining the morphology of the newly formed vasculature. Migrating tip cells probe their environment by extending filopodia and migrate along the VEGF gradient towards regions of higher concentration, a directed motion referred to as chemotaxis. In addition to the soluble isoform of VEGF, the presence of other VEGF isoforms expressing binding sites

M. Bubak et al. (Eds.): ICCS 2008, Part II, LNCS 5102, pp. 167-176, 2008.

(C) Springer-Verlag Berlin Heidelberg 2008 
for the ECM has been identified to significantly influence morphology of capillary network formation 129. These "matrix-bound" VEGF isoforms can be cleaved from the ECM by Matrix MetalloProteinases (MMPs), expressed both by tumors and migrating ECs.

Another component involved in the process of angiogenesis is fibronectin, a glycoprotein distributed in the ECM and at the same time released by migrating tip cells. Fibronectin binds to fibers occupying about $30 \%$ of the ECM. Through interaction with transmembrane receptors located on the EC membrane, fibronectin establishes an adhesive gradient which servers as another migration cue for the ECs. This autocrine signaling pathway, promoting cell-cell and cell-matrix adhesion, accounts for a movement referred to as haptotaxis. In addition to the chemotactic and haptotactic cues, the fibrous structures itself present in the ECM influence cell migration by facilitating movement in fiber direction.

After initial sprouts have extended into the EC for some distance, repeated branching of the tips can be observed. Sprout tips approaching others may fuse and form loops, a process called anastomosis. Along with anastomosis, the formation of lumen within the strands of endothelial cells establishes a network that allows the circulation of blood. In a final stage, the newly formed vessels maturate, establishing a basal lamina and recruit pericytes and smooth muscle cells to stabilize the vessel walls. An overview of the biological processes involved in angiogenesis can be found in 81011 and references therein.

In the following, we propose a mathematical model of sprouting angiogenesis together with the computational methods that implement the equations in $3 \mathrm{D}$. Along with the model, simulation results are shown, underlining the effect of the ECM structure and matrix-bound growth factors on the generated network morphology.

\subsection{Computational Modeling of Angiogenesis}

Computational models of tumor-induced angiogenesis address a limited number of the involved biological processes. The choice of the modeled processes is dictated by the availability of biological data and by the understanding of the underlying biological processes. In the presented model we consider the motion of the ECs as affected by chemical gradients induced by VEGF, haptotactic gradients induced by fibronectin and by the structure of the ECM. We note that the present assumptions may be more pertinent to in-vitro angiogenesis rather than in-vivo angiogenesis which is known to depend on the particular microenvironment [15].

In the present work VEGF appears in soluble and matrix-bound isoforms. The soluble VEGF is released from an implicit tumor source, and diffuses freely through the ECM. The matrix-bound VEGF isoform is randomly distributed and can be cleaved by MMPs released at the sprout tips. Different VEGF isoforms contribute equally to the migration cues of the ECs (see Fig. 1).

Fibronectin is released at sprout tips, establishing a haptotactic gradient for the ECs. In addition, we model the binding of fibronectin to the ECM which 
localises the haptotactic cues. The ECM is explicitly modeled to consist of directed bundles of collagen fibers randomly distributed throughout the domain. A vector field describing the fiber directions modulates the migration velocity of the ECs in the presence of fibers.

A summary of work done in the field of modeling angiogenesis can be found in [10. More recent work includes the influence of blood flow on the process of angiogenesis by Chaplain et al. [6], the model proposed by Sun et al. [14] considering the conductivity of the ECM and a cell based model of angiogenesis by Bauer et al. [2].

The present model is the first, to the best of our knowledge, to include a cleaving mechanism and to present simulations in the presence of both VEGF isoforms. The proposed 3D modeling approach combines the continuum representation [1514 with a cell based approach confined to the migrating tip cells located at the sprouting front. We implement a hybrid approach to represent molecular species by their concentration and migrating EC tip cells by particles. The evolution of molecular species is governed by reaction-diffusion equations that are discretized on the grid while a particle approach is employed in order to model the migrating EC tip cells. The particle and grid descriptions are coupled as the ECs both serve as a source of fibronectin and MMPs and as sink for VEGF (binding to the cell surface receptors). As the tip cells migrate through the ECM following up the chemotactic and haptotactic cues, they "depose" ECs along their way leaving a trail of endothelial cell density on the grid that defines the $3 \mathrm{D}$ vessel structure of the outgrowing sprouts. Filopodia are explicitly modeled to sense chemotactic and haptotactic migration cues, which determine the sprout branching behavior. We report statistics on sprout section length, branching and anastomosis frequency, enabling a quantification of different parametric models and paving the way for future comparisons with experimental works.

\section{Vascular Endothelial Growth Factors}

Matrix-bound VEGF (bVEGF, $\Psi_{\mathrm{b}}$ ) does not diffuse and it is assumed to be locally distributed on the fibers composing the ECM. The bVEGF can be cleaved from the matrix by $\operatorname{MMPs}(\chi)$ released from migrating ECs. Further, ECs express surface receptors that bind VEGF molecules.

$$
\frac{\partial \Psi_{\mathrm{b}}}{\partial t}=-\mathcal{C}\left(\Psi_{\mathrm{b}}, \chi\right)-\mathcal{U}\left(\Psi_{\mathrm{b}}\right)
$$

with the cleaving function:

$$
\mathcal{C}\left(\Psi_{\mathrm{b}}, \chi\right)=\min \left(\Psi_{\mathrm{b}}, v_{b V} \chi \Psi_{\mathrm{b}}\right)
$$

and the cleaving rate $v_{b V}$. The uptake function is given by

$$
\mathcal{U}([\mathrm{C}])=\min \left([\mathrm{C}], v_{V} \sigma\right),
$$

with the endothelial uptake rate of VEGF given by $v_{V}$ and the endothelial cell density $\sigma$. 

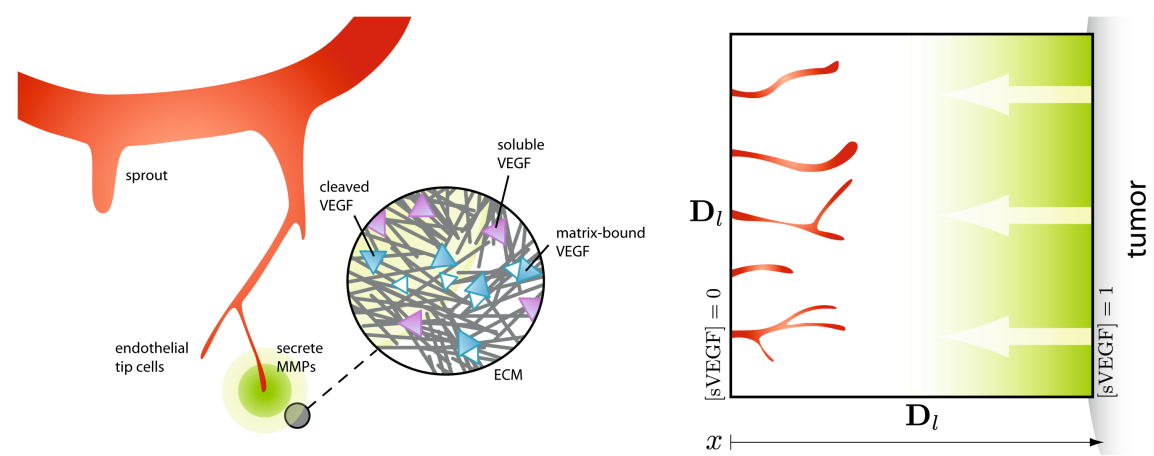

Fig. 1. Left: Conceptual sketch of the different VEGF isoforms present in the ECM. Soluble and cleaved VEGF isoforms freely diffuse through the ECM, Matrix-bound VEGF isoforms stick to the fibrous structures composing the ECM and can be cleaved by MMPs secreted by the sprout tips. Right: Conceptional x-z plane through the computational domain. Five sprout tips are initially placed on the $y-z$ plane lower end of the domain in $\mathrm{x}$ direction, a tumor source of soluble VEGF is modeled at the upper end in $\mathrm{x}$ direction outside the computational domain.

Cleaved bVEGF $\left(\mathrm{cVEGF}, \Psi_{\mathrm{c}}\right)$ and solube VEGF $\left(\mathrm{sVEGF}, \Psi_{\mathrm{s}}\right)$ diffuse through the ECM and are subject to natural decay. Endothelial cell uptake is modeled by the uptake function $\mathcal{U}$.

$$
\frac{\partial \Psi_{\mathrm{c}}}{\partial t}=k_{V} \nabla^{2} \Psi_{\mathrm{c}}+\mathcal{C}\left(\Psi_{\mathrm{b}}, \chi\right)-\mathcal{U}\left(\Psi_{\mathrm{c}}\right)-d_{V} \Psi_{\mathrm{c}} .
$$

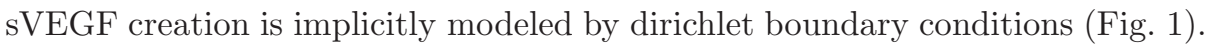

$$
\frac{\partial \Psi_{\mathrm{s}}}{\partial t}=k_{V} \nabla^{2} \Psi_{\mathrm{s}}-\mathcal{U}\left(\Psi_{\mathrm{s}}\right)-d_{V} \Psi_{\mathrm{s}}
$$

\section{Fibronectin}

Fibronectin $(\Phi)$ is released by the migrating ECs depending on the local fibronectin concentration. We consider fibronectin released by ECs binding to integrins located at the EC membrane and to matrix fibers. Fibronectin diffuses through the ECM when not bound to the matrix and is subject to natural decay.

$$
\frac{\partial \Phi}{\partial t}=k_{F} \nabla^{2} \Phi+\gamma_{F} \mathcal{G}\left(F_{t h}, \Phi\right) \Sigma-v_{b F}\left(b F_{t h}-\Phi\right)-d_{F} \Phi,
$$

with creation function

$$
\mathcal{G}\left(C_{t h}, C\right)=\frac{C_{t h}-C}{C_{t h}} .
$$

depending on the local fibronectin concentration and the creation threshold level $F_{t h}$. The rate of fibronection binding to the ECM is given by $v_{b F}$ and limited by $b F_{t h}$ to account for binding site saturation in the ECM. 
Once fibronectin binds to the ECM, further diffusion is inhibited. The matrixbound fibronectin $\left(\Phi_{\mathrm{b}}\right)$ evolution is given by:

$$
\frac{\partial \Phi_{\mathrm{b}}}{\partial t}=v_{b F}\left(b F_{t h}-\Phi\right)-d_{b F} \Phi_{\mathrm{b}}
$$

\subsection{Matrix-MetalloProteinases}

$\operatorname{MMPs}(\chi)$ cleave the bVEGF isoforms from the binding sites in the ECM and are assumed to be released at the migrating ECs depending on the local MMP concentration. The specific release rate is given by $\gamma_{M}$ and $\Sigma$ describes the endothelial tip cell density. MMP release is stopped when the local MMP level approaches the threshold level $M_{t h}$. Upon release by the ECs, MMPs are assumed to diffuse through the ECM and are subject to natural decay.

$$
\frac{\partial \chi}{\partial t}=k_{M} \nabla^{2} \chi+\gamma_{M} \mathcal{G}\left(M_{t h}, \chi\right) \Sigma-d_{M} \chi
$$

\section{Endothelial Cells}

The migration direction of endothelial tip cells is determined by chemotactic and haptotactic cues in the matrix given by VEGF and fibronectin gradients. As the VEGF level increases, EC surface receptors become occupied, attenuating the cells ability to sense the chemotactic cues. The attenuation is represented by a function $\mathcal{W}$. The sprout tip acceleration during migration is defined as :

$$
\mathbf{a}=\alpha\left(E_{\rho}\right) \underline{\mathbf{T}}\left(\mathcal{W}(\Psi) \nabla \Psi+w_{F} \nabla \Phi_{\mathrm{b}}\right),
$$

where

$$
\mathcal{W}(\Psi)=\frac{w_{V}}{1+w_{v 2} \Psi}
$$

and

$$
\Psi=\Psi_{\mathrm{s}}+\Psi_{\mathrm{b}}+\Psi_{\mathrm{c}} .
$$

The presence of fibers $\left(E_{\rho}\right)$ promote mesenchymal motion of the tip cells, thus enhance the migration speed of ECs. In contrast, a very dense matrix slows down the migration speed of the tip cells as characterized by the function:

$$
\alpha\left(E_{\rho}\right)=\left(E_{0}+E_{\rho}\right)\left(E_{1}-E_{\rho}\right) C_{1},
$$

where the threshold $E_{0}$ defines the migration factor in the absence of fibers, $E_{1}$ the maximal fiber density and $C_{1}$ the ECM migration constant . To model the directional cues of the matrix fibers, a tensor $\mathbf{T}$ is introduced acting on the migration velocity.

$$
\{\underline{\mathbf{T}}\}_{i j}=\left(1-\beta\left(E_{\chi}\right)\right)\{1\}_{i j}+\beta\left(E_{\chi}\right) K_{i} K_{j},
$$

with

$$
\beta\left(E_{\chi}\right)=\beta_{K} E_{\chi}
$$


the ECM strength $\beta_{K}$ and $\mathbf{K}$ being the vector field the tensor is applied on. Tip cell particle positions $\mathbf{x}_{p}$ are updated according to:

$$
\frac{\mathbf{x}_{p}}{\partial t}=\mathbf{u}_{p}, \frac{\mathbf{u}_{p}}{\partial t}=\mathbf{a}_{p}-\lambda \mathbf{u}_{p}
$$

with drag coefficient $\lambda$.

The matrix structure may promote diverging migration directions, leading to branching of the endothelial tip cells and creation of new sprouts. In our model, we locate regions of high anisotropy in the migration acceleration direction field by a curvature measure $k$ as proposed in [16].

Branching occurs in locations where the local curvature $k$ exceeds a threshold level $a i_{t h}$. In order to determine the preferred branching direction in 3D, 6 satellite particles are distributed radially around the tip cell particle in a plane perpendicular to the migration direction modeling the extension of filopodia. The velocity field is compared at opposing satellite positions and branching occurs into the directions that diverge the most. ECs are insensitive to branching cues immediately after a branching event has occurred. In order to account for this effect, a sprout threshold age $s a_{t h}$ is introduced. Sprout tips of age smaller than $s a_{t h}$ are not considered for branching. Anastomosis occurs when tip cells fuse either with existing sprouts or with other tip cells.

In order to obtain the endothelial cell density defining the capillary sprouts, at every time step, we interpolate the sprout tip cell density $Q_{p}$ at $\mathbf{x}_{p}$ onto the grid using a 4 th order B-spline kernel $B_{4}$ and add the maximum of the interpolated sprout tips and the $\sigma$ field onto the $\sigma$ field.

$$
\sigma_{i j k}^{n+1}=\max \left(\sigma_{i j k}^{n}, \sum_{p} B_{4}\left(i h-x_{p}\right) B_{4}\left(j h-y_{p}\right) B_{4}\left(k h-z_{p}\right) Q_{p}\right),
$$

with particle weight $Q_{p}$, and mesh size $h$ whereas $n$ denotes the $n$th time step.

\section{ECM}

In the present work the ECM is modeled as a collection of fiber bundles randomly distributed throughout the computational domain. The ECM is represented by three grid-functions: (i) a vector field $\mathbf{K}$ that describes the fiber orientations, (ii) a smooth indicator function $E_{\chi}$, which indicates the presence of fibers at any given point in space, and (iii) a fiber density field $E_{\rho}$, which is used to regulate migration speed.

These fields are constructed in a straightforward manner by generating $N$ random fibers with a given length $\mathbf{f}_{l}$ which is constant for all fibers. These fibers are then put on the grid much like lines are rasterized in computer graphics [4]. In the case of $\mathbf{K}$ the directions are rasterized onto the grid, and in the case of $E_{\chi}$ we tag the grid points at the fiber locations with a value of 1 , resulting in randomly distributed fibers.

The fields $\mathbf{K}$ and $E_{\rho}$ are filtered with a Gaussian filter to achieve a smooth matrix representation. In the case of $E_{\chi}$ this is not possible, so the field is 
constructed by using smoothed fibers. In cases where fibers overlap the maximum value of the two fibers is retained.

\section{Methods}

The time step constraint for diffusion on the molecular species is stricter than for the reaction part. A fractional step algorithm is used to solve the system efficiently. In this algorithm the non linear and linear reaction parts of the equations are solved simultaneously using explicit Euler steps, while linear diffusion is solved implicitly. The systems can be safely decoupled, as EC cell migration occurs on a much smaller time scale than molecular diffusion and steady state can be assumed for the source and sink of the different proteins. VEGF, fibronectin and acceleration gradients for migration velocity and the curvature measure are calculated on the grid using second order finite differences. In order to get the acceleration and curvature on the particle location, Mesh-Particle interpolations are done using the $M_{4}^{\prime}$ kernel [3] while for the interpolation of the sprout tip density onto the grid, Particle-Mesh interpolations employ 4th order B-spline kernel.

\section{Results}

We present results demonstrating the effect of the ECM density on the resulting vessel networks. The computational domain is defined as a cube of $1.5^{3} \mathrm{~mm}$ discretized with a $128^{3}$ uniform grid. The ECM was modeled using random fiber fields, created with five different matrix densities: 15,000 fibers resulting in a volume density of 6\%,30,000 fibers (11\%), 70,000 fibers (26\%), 100,000 fibers $(38 \%)$, and 200,000 fibers $(75 \%)$ (see Fig. 2). The normalized volume density is given by the sum of the fiber density $E_{\rho}$ over all grid points divided by the number of grid points. For each density value we performed 128 simulations with a different random seed for the fiber placement.

Comparing the number of branches found in the computational domain at simulation time $T=25.0$ corresponding to 10.9 days in physical time, (Fig. 3) we find a logarithmic increase of the number of branches for linearly increasing fiber density. Examples of the structure of the corresponding vessel networks are depicted in Fig. 2: in very low density ECMs, hardly any branching occurs, while in very dense ECMs the EC sprouts branch very often. In the $75 \%$ density case the fiber density is high enough to impair the migration which leads to shorter capillary networks (Fig. 2 E). In Fig. 4 we depict the evolution of the vascular network in the presence of initially distributed pockets of bVEGF. The bVEGF pockets are cleaved by MMPs (not shown) as the sprouts approach the VEGF source at the far end of the domain. The vessels grow in diameter by application of a post-processing vessel maturation method(not described in this work). 


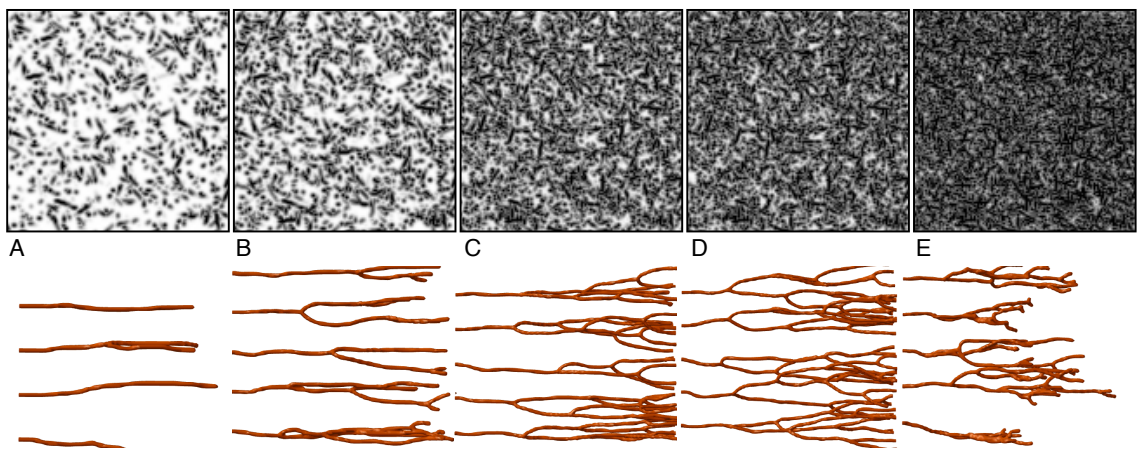

Fig. 2. Top: Slice of the ECM field for five different densities: A 6\%, B 11\%, C 26\%, D $38 \%$, and E 75\%. Bottom: Capillary networks for the different ECM densities.

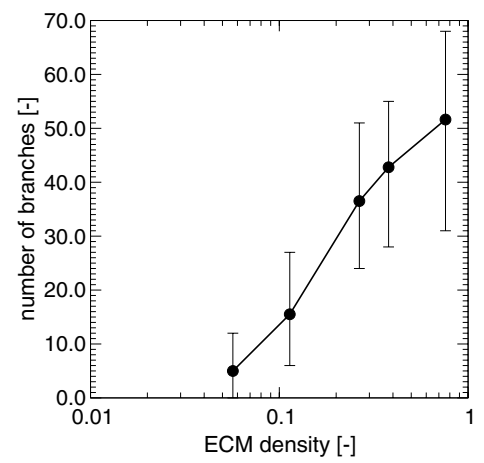

Fig. 3. Influence of the matrix density on the number of branches of the vessel network (error bars represent $\min / \max$ of data)

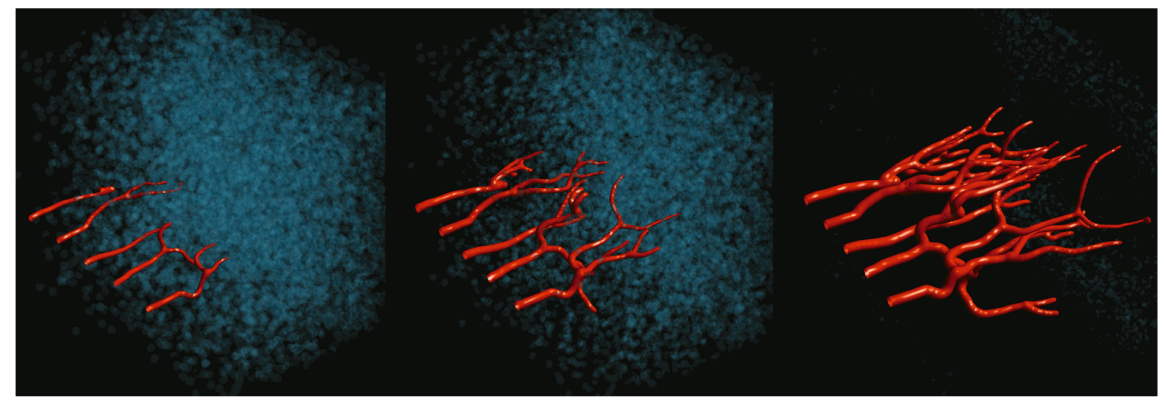

Fig. 4. Capillary network evolution in the presence of bVEGF pockets. bVEGF is cleaved by MMPs during the course of the simulation. 


\section{Conclusions}

The present work describes the first, to the best of our knowledge, simulations of $3 \mathrm{D}$ sprouting angiogenesis that incorporate effects of the extracellular matrix structure on the vessel morphology and considers both soluble and matrix-bound growth factor isoforms. The method is formulated as a generalized hybrid particle method and is implemented in the context of a parallel framework (PPM) 13. This aspect of the method renders it scalable to massively parallel computer architectures, a crucial aspect for the study of angiogenesis at macroscopic scales and integrative models of vascular tumor growth. Efficient Particle to Mesh and Mesh to Particle interpolation schemes provide a straightforward way of coupling the two levels of representation.

The presented simulations of sprouting angiogenesis have shown that the structure and density of the ECM has a direct effect on the morphology, expansion speed and number of branches observed in computationally grown vessel networks. The simulations reflect the influence of the extracellular matrix composition on endothelial cell migration and network formation corresponding to observations made by 7 .

With the number of branches depending on the matrix structure and the presence and level of matrix-bound VEGF isoforms, this model may be easier to tune against experiments compared to branching probabilities that most individual-based methods employ.

Limitations of the current model are related to the explicit definition of tip cells restricting the formation of new sprout tips to predefined locations on the initial vasculature. Formulation of a tip cell selection method combined with cell type specific migration and proliferation rules are the subject of current work. The integration of the present framework in studies of tumor induced angiogenesis is a subject of coordinated investigations with experimental groups.

\section{References}

1. Anderson, A.R.A., Chaplain, M.A.J.: Continuous and Discrete Mathematical Models of Tumor-Induced Angiogenesis. Bull. Math. Biol. 60(5), 857-899 (1998)

2. Bauer, A.L., Jackson, T.L., Jiang, Y.: A Cell-Based Model Exhibiting Branching and Anastomosis During Tumor-Induced Angiogenesis. Biophys. J. 92, 3105-3121 (2007)

3. Bergdorf, M., Koumoutsakos, P.: A Lagrangian Particle-Wavelet Method. Multiscale Model. and Simul. 5(3), 980-995 (2006)

4. Bresenham, J.E.: Algorithm for Computer Control of a Digital Plotter. IBM Syst. J. 4(1), 25-30 (1965)

5. Chaplain, M.A.: Mathematical Modelling of Angiogenesis. J. Neurooncol. 50(1-2), 37-51 (2000)

6. Chaplain, M.A.J., McDougall, S.R., Anderson, A.R.A.: Mathematical Modeling of Tumor-Induced Angiogenesis. Annu. Rev. Biomed. Eng. 8, 233-257 (2006)

7. Davis, E.G., Senger, D.R.: Endothelial Extracellular Matrix: Biosynthesis, Remodeling, and Functions During Vascular Morphogenesis and Neovessel Stabilization. Circ. Res. 97(11), 1093-1107 (2005) 
8. Folkman, J.: Angiogenesis: an Organizing Principle for Drug Discovery? Nat. Rev. Drug Discov. 6(4), 273-286 (2007)

9. Lee, S., Jilani, S.M., Nikolova, G.V., Carpizo, D., Iruela-Arispe, M.L.: Processing of VEGF-A by Matrix Metalloproteinases Regulates Bioavailability and Vascular Patterning in Tumors. J. Cell Biol. 169(4), 681-691 (2005)

10. Mantzaris, N., Webb, S., Othmer, H.: Mathematical Modeling of Tumor-Induced Angiogenesis. J. Math. Biol. 49, 111-187 (2004)

11. Paweletz, N., Knierim, M.: Tumor-Related Angiogenesis. Crit. Rev. in Oncol. Hematol. 9(3), 197-242 (1989)

12. Ruhrberg, C., Gerhardt, H., Golding, M., Watson, R., Ioannidou, S., Fujisawa, H., Betsholtz, C., Shima, D.T.: Spatially Restricted Patterning Cues Provided by Heparin-Binding VEGF-A Control Blood Vessel Branching Morphogenesis. Genes Dev. 16(20), 1684-2698 (2002)

13. Sbalzarini, I.F., Walther, J.H., Bergdorf, M., Hieber, S.E., Kotsalis, E.M., Koumoutsakos, P.: PPM - a Highly Efficient Parallel Particle-Mesh Library. J. Comput. Phys. 215(2), 566-588 (2006)

14. Sun, S., Wheeler, M.F., Obeyesekere, M., Patrick Jr., C.W.: A Deterministic Model of Growth Factor-Induced Angiogenesis. Bull. Math. Biol. 67, 313-337 (2005)

15. Sung, S.Y., Hsieh, C.L., Wu, D., Chung, L.W.K., Johnstone, P.A.S.: Tumor Microenvironment Promotes Cancer Progression, Metastasis, and Therapeutic Resistance. Curr.Prob. Cancer 31(2), 36-100 (2007)

16. Weinkauf, T., Theisel, H.: Curvature Measures of 3d Vector Fields and their Applications. J. WSCG. 10, 507-514 (2002) 